\title{
Topological submanifolds and homology classes of a topological manifold
}

By

\author{
Masahisa AdAchi*)
}

(Received May 28, 1971)

This note is devoted to the problem of the realization of homology classes of a topological manifold by topological submanifolds. Firstly the $C^{\infty}$-case of this problem was studied by $\mathrm{R}$. Thom [6], and secondly the $P L$-case in $[1],[2]$.

The present study is founded on the Kirby-Siebenmann's transversality theorem [3]. We shall apply R. Thom's method [6] to topological manifolds.

The author is grateful to Professor Y. Saito and Mr. T. Matumoto for their kind criticisms.

\section{Statement of the results}

We shall obtain the following results.

i) Homology classes mod 2 .

Theorem 1. Let $V^{n}$ be a closed topological manifold of dimension $n$, and $n \neq 4$. Then the following homology classes mod 2 are realizable by topological submanifolds which have normal vector bundles in $V^{n}$ :
(a) $H_{n-1}\left(V^{n}, \mathbf{Z}_{2}\right), \quad$ for $n \neq 5, n \geqq 1$;
(b) $H_{n-2}\left(V^{n}, \mathbf{Z}_{2}\right), \quad$ for $2 \leqq n<6$; 
(c) $H_{n-3}\left(V^{n}, \mathbf{Z}_{2}\right), \quad$ for $3 \leqq n<7$;

(d) $H_{i}\left(V^{n}, \mathbf{Z}_{2}\right), \quad$ for $i \leqq n / 2, i \neq 4$, and all $n \geqq 1$.

ii) Integral homology classes.

Theorem 2. Let $V^{n}$ be a closed orientable topological manifold of dimension $n$ and $n \neq 4$. Then the following integral homology classes are realizable by oriented topological submanifolds which have normal vector bundles in $V^{n}$ :

(a) $H_{n-1}\left(V^{n}, \mathbf{Z}\right), \quad$ for $n \neq 5, n \geqq 1$;

(b) $H_{n-2}\left(V^{n}, \mathbf{Z}\right), \quad$ for $n \neq 6, n \geqq 2$;

(c) $H_{i}\left(V^{n}, \mathbf{Z}\right), \quad$ for $i \leqq 5, i \neq 4$ and for all $n \geqq 1$.

Remark 1. The normal bundle of an orientable submanifold in an orientable manifold is a priori orientable.

Remark 2. A topological submanifold which has a normal vector bundle is clearly a locally flatly embedded submanifold.

These results are quite in parallel to those of the $C^{\infty}$-case in Thom [6].

\section{Generalities}

We shall work in the category of topological spaces and continuous maps.

Let $V^{n}$ be a topological manifold of dimension $n$. Then we shall say that $W^{p}$ is a topological submanifold of dimension $p$, if $W^{p}$ is a closed topological manifold of dimension $p$ and a topological subspace of $V^{n}$.

Let $V^{n}$ be a closed topological manifold of dimension $n$. Let $W^{p}$ be a topological submanifold of $V^{n}$ of dimension $p$. The inclusion map $i: W^{p} \rightarrow V^{n}$ induces the homomorphism

$$
i_{*}: H_{p}\left(W^{p}, \mathbf{Z}_{2}\right) \rightarrow H_{p}\left(V^{n}, \mathbf{Z}_{2}\right) .
$$

Let $z \in H\left(V^{n}, \mathbf{Z}_{2}\right)$ be the image by $i_{*}$ of the fundamental class $w$ of 
the topological manifold $W^{p}$. Then we say that the homology class $z$ is realized by the topological submanifold $W^{p}$. Let $V^{n}$ be orientable, and $W^{p}$ be an oriented topological submanifold of dimension $p$. Then the inclusion map $i_{*}: W^{p} \rightarrow V^{n}$ induces the homomorphism

$$
i_{*}: H_{p}\left(W^{p}, \mathbf{Z}\right) \rightarrow H_{p}\left(V^{n}, \mathbf{Z}\right) .
$$

Let $z \in H\left(V^{n}, \mathbf{Z}\right)$ be the image by $i_{*}$ of the fundamental class $w$ of the oriented topological manifold $W^{p}$. Then we say that the homology class $z$ is realized by the oriented topological submanifold $W^{D}$.

Here the following questions are considered: Let a homology class $z \bmod 2$ of a closed topological manifold $V^{n}$ be given. Is it realizable by a topological submanifold? ; Let an integral homology class $z$ of a closed orientable topological manifold $V^{n}$ be given. Is it realizable by an oriented topological submanifold?

Following J. Kister [4], let $\mathscr{H}_{0}(k)$ be the space of all homeomorphisms of the Euclidean $k$-space $\mathbf{R}^{k}$ onto itself preserving the origin 0 with compact-open topology. Then $\mathscr{H}_{0}(k)$ is a topological group with respect to the composition of maps. Let $S \mathscr{H}_{0}(k)$ be the subgroup of $\mathscr{H}_{0}(k)$ of those elements that preserve orientation.

By an $\mathbf{R}^{k}$-bundle we shall mean a fibre bundle whose fibre is the Euclidean $k$-space $\mathbf{R}^{k}$ and structure group is $\mathscr{H}_{0}(k)$. Let

$\xi=\left\{E(\xi), \pi_{\xi}, B(\xi), \mathbf{R}^{k}, \mathscr{H}_{0}(k)\right\}$ be an $\mathbf{R}^{k}$-bundle. Then $\xi$ has the 0 -cross-section

$$
i_{\xi}: B(\xi) \rightarrow E(\xi)
$$

By an orientable $\mathbf{R}^{k}$-bundle we shall mean a fibre bundle whose fibre is the Euclidean $k$-space $\mathbf{R}^{k}$ and structure group is $S \mathscr{H}_{0}(k)$. The orthogonal group $O(k)$ can be canonically considered as a topological subgroup of $\mathscr{H}_{0}(k)$. Therefore, we can canonically consider a vector bundle of dimension $k$ to be an $\mathbf{R}^{k}$-bundle.

Let $V^{n}$ be a closed topological manifold and $W^{k}$ be a topological submanifold of $V^{n}$. Then by a normal $\mathbf{R}^{n-k}$-bundle of $W^{k}$ in $V^{n}$, we shall mean an $R^{n-k}$-bundle $\nu=\left\{E(\nu), \pi_{\nu}, B(\nu), \mathbf{R}^{n-k}, \mathscr{H}_{0}(n-k)\right\}$ whose 
base space $B(\nu)$ is $W^{k}$ and total space $E(\nu)$ is a neighborhood of $W^{k}$ in $V^{n}$.

Let $V^{n}$ be a closed topological manifold and $W^{k}$ a topological submanifold of $V^{n}$. Then by a normal vector bundle of $W^{k}$ in $V^{n}$, we shall mean a vector bundle $\nu=\left\{E(\nu), \pi_{\nu}, B(\nu), \mathbf{R}^{n-k}, O(n-k)\right\}$ whose base space $B(\nu)$ is $W^{k}$ and total space $E(\nu)$ is a neighborhood of $W^{k}$ in $V^{n}$.

\section{Transversality theorem of Kirby-Siebenmann}

Let $\xi=\left\{E(\xi), \pi_{\xi}, B(\xi), \mathbf{R}^{n}, \mathscr{H}_{0}(n)\right\}$ be an $\mathbf{R}^{n}$-bundle and $M^{m}$ be a topological $m$-manifold. By considering the zero-cross-section, we can consider $B(\xi) \subset E(\xi)$. A continuous map $f: M^{m} \rightarrow E(\xi)$ is called to be transverse to $B(\xi)$, if $P=f^{-1}(B(\xi))$ is an $(m-n)$-dimensional topological submanifold with normal $\mathbf{R}^{n}$-bundle $\nu$ in $M^{m}$ and $\nu$ is isomorphic to the induced bundle $(f \mid P)^{*}$.

Kirby-Siebenmann [3] have proved the following transversality theorem.

Theorem 3. Let $\xi=\left\{E(\xi), \pi_{\xi}, B(\xi), \mathbf{R}^{n}, \mathscr{H}_{0}(n)\right\}$ be an $\mathbf{R}^{n}$-bundle and $M^{m}$ be a topological m-manifold. Let $f: M^{m} \rightarrow E(\xi)$ be a continuous map. Then, if $m \neq 4, m-n \neq 4, f$ is homotopic to a map $f_{1}$ which is transverse to $B(\xi)$. If $f$ is transverse to $B(\xi)$ near a closed set $C \subset M^{m}$, then the homotopy equals to $f$ near $C$.

\section{Fundamental theorem.}

Definition. We say that a cohomology class $u \in H^{k}\left(A, \mathbf{Z}_{2}\right)$ of a space $A$ is $O(k)$-realizable, if there exists a mapping $f: A \rightarrow M O(k)$ such that $u$ is the image, for the homomorphism $f^{*}$ induced by $f$, of the fundamental class $U_{o(k)}$ of the Thom complex $M O(k)$. We say that an integral cohomology class $u \in H^{k}(A, \mathbf{Z})$ of a space $A$ is $S O(k)$-realizable, if there exists a mapping $f: A \rightarrow M S O(k)$ such that $u$ is the image, for the homomorphism $f^{*}$ induced by $f$, of the fundamental class $U_{S O(k)}$ of the Thom complex $M S O(k)$. 
Then we have the following fundamental theorem.

Theorem 4. Let $V^{n}$ be a closed topological manifold of dimension $n$. Suppose that $n \neq 4, n-k \neq 4$.

(a) In order that homology class $z \in H_{n-k}\left(V^{n}, \mathbf{Z}_{2}\right), k>0$ can be realized by a topological submanifold $W^{n-k}$ which has a normal vector bundle in $V^{n}$, it is necessary and sufficient that the cohomology class $u \in H^{k}\left(V^{n}, \mathbf{Z}_{2}\right)$, corresponding to $z$ be the Poincaré duality, is $O(k)$. realizable.

(b) Let $V^{n}$ be orientable. In order that an integral homology class $z \in H_{n-k}\left(V^{n}, \mathbf{Z}\right), k>0$, can be realized by an oriented topological submanifold $W^{n-k}$ which has a normal vector bundle in $V^{n}$, it is necessary and sufficient that the cohomology class $u \in H^{k}\left(V^{n}, \mathbf{Z}\right)$, corresponding to $z$ by the Poincaré duality, is $S O(k)$-realizable.

Proof. We shall prove the case (a) of the theorem. The case (b) can be proved quite in parallel with the case (a).

(i) The condition is necessary. Suppose that there exists a topological submanifold $W^{n-k}$ in $V^{n}$ which realizes the homology class $z$, and has a normal vector bundle $\nu$ in $V^{n}$ :

$$
\nu=\left\{E(\nu), \pi_{\nu}, B(\nu), \mathbf{R}^{k}, O(k)\right\}, B(\nu)=W^{n-k} .
$$

Let $N$ be the total space of the associated $k$-disk bundle $\nu_{D}$ of $\nu$, and $T$ be the total space of the associated $S^{k-1}$-bundle $\nu_{S}$ of $\nu$; namely $T$ is the boundary of $N$. Then we can consider

$$
W^{n-k}=B(\nu) \subset N \subset E(\nu) \subset V^{n} .
$$

Let

$$
\nu_{D}=\left\{N, \pi_{D}, W^{n-k}, D^{k}, 0(k)\right\} .
$$

Then $\nu_{D}$ is induced from the universal $D^{k}$-bundle $\nu_{D}^{k}=\left\{A_{0(k)}, \pi_{k}, B_{0(k)}\right.$, $\left.D^{k}, O(k)\right\}$ by a continuous mapping $g: W^{n-k} \rightarrow B_{0(k)}$. Therefore, there exists a bundle map $\tilde{g}$ which induces $g$ : 


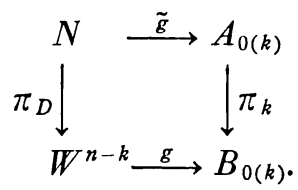

Let $\gamma_{s}^{k}=\left\{E_{0(k)}, \pi_{k}, B_{0(k)}, S^{k-1}, O(k)\right\}$ be the universal $S^{k-1}$-bundle. Then the restriction of $\tilde{g}$ on the boundary $T$ of $N$ maps $T$ in the boundary $E_{0(k)}$ of $A_{0(k)}$. Therefore, we have the following commutative diagram:

$$
\begin{aligned}
& H^{k}\left(N, T ; \mathbf{Z}_{2}\right) \stackrel{\tilde{g}^{*}}{\stackrel{k}{g}} H^{k}\left(A_{0(k)}, E_{0(k)} ; \mathbf{Z}_{2}\right) \\
& \left.\varphi_{\nu}^{* \uparrow}\right\rangle\|\quad \imath\| \uparrow \varphi_{0(k)}^{*} \\
& \left.H^{0}\left(W^{n-k}, \mathbf{Z}_{2}\right) \stackrel{g^{*}}{\stackrel{0}{ }} H^{(} B_{0(k)}, \mathbf{Z}_{2}\right),
\end{aligned}
$$

where $\varphi_{\nu}^{*}$ and $\varphi_{0(k)}^{*}$ are Thom isomorphisms.

On the other hand, we have the following canonical homomorphism $j_{*}=j^{*} \circ \alpha$ :

$$
j_{*}: H^{k}\left(N, T ; \mathbf{Z}_{2}\right) \underset{\cong}{\stackrel{\alpha}{\cong}} H^{k}\left(V^{n}, V^{n} \text {-int } N ; \mathbf{Z}_{2}\right) \stackrel{j^{*}}{\longrightarrow} H^{k}\left(V^{n}, \mathbf{Z}_{2}\right),
$$

where $\alpha$ is the excision isomorohism and $j^{*}$ is the relativization. We know that in the open manifold $N^{\prime}=N-T=$ int $N$, the class $\varphi_{\nu}^{*}(\omega)$ corresponds, by the Poincaré duality, to the fundamental homology class $w$ of the base $W^{n-k}$, where is $\omega$ the unit of the cohomology ring $H^{*}\left(W^{n-k}, \mathbf{Z}_{2}\right)$ (cf. Thom [5], Théorème I.8). Consequently, the class $j_{*} \circ \varphi_{\nu}^{*}(\omega) \in H^{k}\left(V^{n}, \mathbf{Z}_{2}\right)$ is the class $u$ corresponding to $z$.

Let us denote by $h: A_{0(k)} \rightarrow M O(k)$ the mapping obtained by identifying to a point a the boundary $E_{0(k)}$ of $A_{0(k)}$.

The composite mapping $h \circ \tilde{g}$ maps the boundary $T$ of $N$ on the point $a$. Consequently, the mapping $h \circ \tilde{g}$ can be extended on the whole manifold $V^{n}$; it suffices to map the complement $V^{n}-N$ to the point a. Thus we have defined a mapping $f$ of $V^{n}$ into $M O(k)$, for which we have

$$
f^{*}\left(U_{0(k)}\right)=f^{* \circ \varphi_{0(k)}^{*}}\left(\omega_{0(k)}\right)=j_{*} \circ \varphi_{\nu}^{*}(\omega)
$$


by the commutative diagram (1), where $\omega_{0(k)}$ is the unit of the cohomology ring $H^{*}\left(B_{0(k)}, \mathbf{Z}_{2}\right)$, and

$$
j_{*} \circ \varphi_{\nu}^{*}(\omega)=D_{V} \circ i_{*} \circ D_{W}(\omega)=u,
$$

where $D_{V}, D_{W}$ are the Poincaré duality of $V^{n}, W^{n-k}$, respectively, and $i: W^{n-k} \rightarrow V^{n}$ is the inclusion.

(ii) The condition is sufficient. Suppose that there exists a mapping $f$ of $V^{n}$ into $M O(k)$, such that $f^{*}\left(U_{0(k)}\right)=u$. The space $M O(k)$, with the exceptional point a deprived, can be considered as an $\mathbf{R}^{k}$-bundle $\gamma_{R}^{k}=\left\{A_{0(k)}^{\prime}, \pi^{\prime}, B_{0(k)}, R^{k}, O(k)\right\}$ over $B_{0(k)}$. The restriction of $f$ on the complement $V^{n}-f^{-1}(a)$ is a mapping of the topological $n$-manifold $V^{n}$ $-f^{-1}(a)$ into an $\mathbf{R}^{k}$-bundle $A_{0(k)}^{\prime}$. Let $\gamma_{D}^{k}=\left\{A_{0(K)}, \pi_{D}, B_{0(k)}, D^{k}, 0(k)\right\}$ be $D^{k}$-bundle associated to $\gamma_{R}^{k}$. Then we can consider $B_{0(k)} \subset A_{0(k)} \subset$ $A_{0(k)}^{\prime}$. Let $C=f^{-1}\left(A_{0(k)}^{\prime}-\operatorname{int} A_{0(k)}\right)$. Then $C$ is a closed set in $V^{n}-$ $f^{-1}(a)$ and $f$ restricted on a neighborhood $U$ of $C$ is $t$-regular on $B_{0(k)}$. By Kirby-Siebenmann's transversality theorem (Theorem 3 ), we obtain a new mapping $f_{1}$ of $V^{n}$-int $f^{-1}(a)$ into the $\mathbf{R}^{k}$-bundle $A_{0(k)}^{\prime}$, which is $t$-regular on $B_{0(k)}$, and homotopic to $f \mid\left(V^{n}-f^{-1}(a)\right)$. Moreover, we can take $f_{1}$ on the neighborhood $U$ of $C$ to be $f \mid\left(V^{n}-f^{-1}(a)\right)$ on $U$. Therefore, we can extend the mapping $f_{1}$ on $V^{n}$ :

$$
f_{1}: V^{n} \rightarrow M O(k),
$$

and $f_{1}$ is homotopic to the given mapping $f$. Consequently, we have $u=f^{*}\left(U_{0(k)}\right)=f_{1}^{*}\left(U_{0(k)}\right)$. On the other hand, by the definition of $t$-regularity (cf. $\S 4$ ), we obtain that $f_{1}^{-1}\left(B_{0(k)}\right)$ is a topological submanifold $W^{n-k}$ and this has a normal $\mathbf{R}^{k}$-bundle $\nu$ of $W^{n-k}$ in $V^{n}$ which is induced from $\gamma_{R}^{k}$ by $f_{1} \mid W^{n-k}$. However, the structure group of $\gamma_{R}^{k}$ is $O(k)$, therefore, the structure group of the normal $\mathbf{R}^{k}$-bundle $\nu$ can be reduced to $O(k)$. Thus we can consider that the submanifold $W^{n-k}$ has a normal vector bundle $\nu$ in $V^{n}$.

Let us denote by $\varphi_{\nu}^{*}$ the Thom isomorphism of the normal vector bundle $\nu$. Then the class $u=f^{*}\left(U_{0(k)}\right)=f_{1}^{*}\left(U_{0(k)}\right)$ is $j_{*} \circ \varphi_{\nu}^{*}(\omega)$. As we have seen in (i), this proves that $u$ corresponds by the Poincaré 
duality to the fundamental class $w$ of $W^{n-k}$.

\section{Proof of Theorems.}

We know that $R$. Thom studied on the homotopy types of Thom complexes $M O(k)$ and $M S O(k)$ (cf. Thom [6], Chapitre II). By these results and the fundamental theorem (Theorem 4), we have Theorem 1 and Theorem 2.

\section{Mathematical Institute, KYOTO UNIVERSITY}

\section{References}

[1] M. Adachi, $P L$-submanifolds and homology classes of a $P L$-manifold, Nagoya Math. J., 29 (1967), 69-74.

[2] M. Adachi, $P L$-submanifolds and homology classes of a $P L$-manifold II, J. Math. Kyoto Univ., 7 (1967), 245-250.

[3] R. Kirby-L. Siebenmann, Some theorems on topological manifolds, Lecture Notes in Math., Springer, 197 (1971), 1-7.

[4] J. Kister, Microbundles are fibre bundles, Ann. of Math., 80 (1964), 190-199.

[5] R. Thom, Espaces fibrés en sphères et carrés de Steenrod, Ann. Sci. Ecole Norm. Sup., 69 (1952), 109-182.

[6] R. Thom, Quelques propriétés globales des variétés différentiables, Comment. Math. Helv., 28 (1954), 17-86. 